\title{
INTOLERÂNCIA À LACTOSE: MUDANÇA DE PARADIGMAS COM A BIOLOGIA MOLECULAR
}

\author{
Rejane Mattar ${ }^{1 *}$, Daniel Ferraz de Campos Mazo²
}

Trabalho realizado no Departamento de Gastroenterologia da Faculdade de Medicina da Universidade de São Paulo, São Paulo, SP

\author{
*Correspondência: \\ Av. Dr. Enéas de Carvalho \\ Aguiar $255,9^{\circ}$ andar, sala \\ 9159 \\ Cerqueira Cesar \\ CEP: 05403-000 \\ São Paulo - SP \\ rejane-mattar@ig.com.br
}

\begin{abstract}
RESUMO
Na maioria dos mamíferos a atividade da enzima lactase diminui na parede intestinal após o desmame, caracterizando a hipolactasia primária que provoca sintomas de intolerância à lactose. A intensidade dos sintomas de distensão, flatulência, dor abdominal e diarreia variam, dependendo da quantidade de lactose ingerida, e aumentam com o passar da idade. A hipolactasia é determinada geneticamente, porém uma mutação ocorreu para que fizesse parte da humanidade tolerar o leite na idade adulta. O diagnóstico é feito por teste de tolerância, empregando a lactose como desafio. Com a descoberta dos finlandeses do polimorfismo associado com a persistência da lactase, principalmente no norte da Europa, o exame genético passou a ser outra ferramenta diagnóstica mais confortável para o intolerante. No Brasil, $43 \%$ dos brancos e dos mulatos têm alelo de persistência da lactase, sendo a hipolactasia mais frequente entre os negros e japoneses. Entretanto, na prática clínica indivíduos com hipolactasia podem ser orientados a consumir alguns derivados do leite e alimentos contendo lactose sem apresentar sintomas de intolerância, enquanto que outros terão que fazer restrição de lactose na dieta.
\end{abstract}

UnItermos: Intolerância à lactose. Fatores de lactose. Epidemiologia. Biologia molecular.

\section{INTRODUÇÃO \\ Conceito}

Má absorção ou má digestão de lactose é a diminuição na capacidade de hidrolisar a lactose, que é resultante da hipolactasia. A hipolactasia significa diminuição da atividade de enzima lactase na mucosa do intestino delgado ${ }^{1}$, também denominada recentemente de "lactase não persistente" 2 . 0 aparecimento de sintomas abdominais por má absorção de lactose caracteriza a intolerância à lactose. A má absorção de lactose nem sempre provoca sintomas de intolerância à lactose ${ }^{1}$. Após o desmame, ocorre uma redução geneticamente programada e irreversível da atividade da lactase na maioria das populações do mundo, cujo mecanismo é desconhecido, resultando em má absorção primária de lactose ${ }^{3}$. Porém, a hipolactasia também pode ser secundária a doenças que causem dano na borda em escova da mucosa do intestino delgado ou que aumentem significativamente o tempo de trânsito intestinal, como nas enterites infecciosas, giardíase ${ }^{4}$, doença celíaca ${ }^{5}$, doença inflamatória intestinal (especialmente doença de Crohn), enterites induzidas por drogas ou radiação, doença diverticular do cólon ${ }^{6}$ e anemia (estudo em ratos, mostrando diminuição na expressão

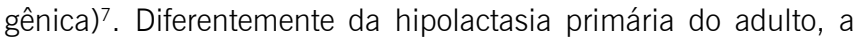
hipolactasia secundária é transitória e reversível${ }^{8}$.

\section{Enzima lactase-florizina hidrolase da família 1 das glicosil hidrolases}

A estrutura primária traduzida da enzima lactase-florizina hidrolase foi deduzida a partir de sequências de CDNA, apresentando 1927 aminoácidos (NP_002290.2- número de acesso no NCBI), sendo que as duas atividades de lactase e florizina hidrolase (glicosilceramidase) se localizam no mesmo polipeptídeo ${ }^{9}$. A estrutura inicial tem peso molecular de 215000 e, após ser processada provavelmente no complexo de Golgi, o peso molecular cai para 160000 , se ancorando na membrana da borda em escova do enterócito ${ }^{10}$, porém em gel filtração apresenta 320000 de peso molecular, sugerindo a formação de dímeros ${ }^{11}$. É ancorada pelo segmento hidrofóbico próximo à região carboxi-terminal, tendo pequena região hidrofílica carboxiterminal no citosol, com provável orientação amino-terminal fora e carboxi-terminal dentro da célula?

\section{Gene $L C T$ que codifica lactase}

Empregando sonda de CDNA humano do gene da lactase ( $L C T$, NG 008104 - número de acesso no NCBI) em Southern blots, foi verificado que o mesmo está localizado no cromossomo $2 q_{2} 1^{12}$. 0 gene $L C T$ compreende 17 exons em $49 \mathrm{~kb}$, sendo traduzido em transcrito (RNAm) de $6 \mathrm{~kb}$. A sequência de DNA do gene e do cDNA

1. Doutorado - Médica Assistente Doutora do Serviço de Gastroenterologia Clínica do Hospital das Clínicas da Faculdade de Medicina da Universidade de São Paulo FMUSP, Responsável pelo Laboratório de Provas Funcionais do Aparelho Digestivo e Professora Colaboradora do Departamento de Gastroenterologia da FMUSP, São Paulo, SP

2. Doutorando - Médico Gastroenterologista e Hepatologista Assistente do Departamento de Gastroenterologia da Faculdade de Medicina da Universidade de São Paulo - FMUSP, São Paulo, SP 
(tradução de RNAm em cDNA) que codifica a enzima lactase em indivíduos com hipolactasia, e naqueles com persistência da enzima lactase na idade adulta foi semelhante, concluindo que ambos os grupos apresentam lactases idênticas, mostrando que a diferença entre os dois grupos não estava na sequência da enzima ${ }^{13}$.

\section{A descoberta genética na Finlândia revolucionou a intolerância à lactose primária do adulto}

Enattah et al. (2002)14, usando desequilíbrio de ligação e análise de haplótipo de nove famílias finlandesas com hipolactasia, detectou dois polimorfismos LCT-13910C $>T$ e LCT-22018G $>$ A acima do lócus $L C T$, sendo que a variante polimórfica LCT-13910C >T apresentou associação completa à não persistência da enzima lactase em 236 indivíduos de quatro diferentes populações (França, América do Norte, Coreia e Alemanha). Os indivíduos com o genótipo CC apresentaram sintomas de intolerância à lactose, e aqueles com os genótipos CT e TT toleraram a lactose ${ }^{14}$, confirmando os achados publicados em 1973 por Sahi et al. de que a intolerância à lactose primária do adulto é traço recessivo. ${ }^{15}$

Estudos posteriores no Brasil ${ }^{16,17,18}$ e em outros países ${ }^{19-26}$ confirmaram a associação desse polimorfismo com a intolerância e tolerância à lactose, exceto na África. ${ }^{27,28}$ Essa região $L C T-13910 C>T$ funcionou in vitro como elemento cis capaz de aumentar diferentemente a atividade de transcrição do promotor do gene da lactase ${ }^{29}$ e se associou significantemente com maior expressão de RNAm do gene $L C T$ na mucosa intestinal dos indivíduos com alelo de persistência da lactase, LCT-13910C > T e LCT-22018G >A, em relação aos que têm genótipo de hipolactasia, sugerindo que a ação seja em nível de regulação de transcrição do gene $L C T^{30}$.

Esses polimorfismos estão situados em regiões intrônicas do gene MCM6 (manutenção de minicromossomo tipo 6); portanto, não interferem com a função deste gene. O polimorfismo LCT13910C > T está localizado no intron 13, o LCT-22018G >A no intron $9^{14}$. As variantes descritas na África foram a $L C T$ 22018G $>$ A no intron 9, LCT-14010G >C, LCT-13915T>G , LCT-13910C > T (traço europeu encontrado em menos de $14 \%$ dos Fulanis e Hausas de Camarões), e LCT-13907C > G no intron $13^{28}$. Esses alelos foram denominados de alelos de enzima lactase persistente, ou de lactasia persistente ${ }^{14}$.

O aparecimento desses alelos como parte do genoma humano suscitou diversas hipóteses. A primeira sugere que eram raros até o aparecimento do hábito de consumir leite não fermentado e laticínios no início do período Neolítico, aumentando a frequência por seleção natural. A outra hipótese seria a da existência prévia dos alelos, favorecendo a aquisição do hábito de consumir produtos lácteos ${ }^{31}$. Análise de DNA mitocondrial de oito esqueletos do período Neolítico e um do Mesolítico mostrou ausência do alelo de lactase persistente, favorecendo a primeira hipótese que tenham aparecido mais tarde com a introdução do consumo de laticínios ${ }^{31}$. Contudo, o estudo de variação de microsatélite intra-alélica de 794 cromossomos de Portugal, Itália, Camarões (Fulanis), São Tomé e Moçambique mostrou que o alelo LCT-13910T se originou na Eurásia antes do período Neolítico e após a emergência do homem moderno para África. Isto sugere que as variantes alélicas LCT-13910T e LCT-22018 se originaram de diferentes mutações, independentemente, na Europa e na África ${ }^{32}$, de duas populações que sofreram forte pressão da seleção natural pela dependência do consumo de leite e de seus derivados na idade adulta para sobreviver. ${ }^{33}$ Entretanto, a grande variabilidade nos genes que codificam as seis principais proteínas do leite de vaca observada no gado de regiões de pecuária do período Neolítico (cerca de 5000 anos atrás) em relação aos demais gados europeus coincidiu com a tolerância à lactose dos europeus de hoje. Portanto, a co-evolução de gene e cultura pode ter ocorrido também entre o gado e os seres humanos ${ }^{34}$.

\section{Epidemiologia}

Os estudos epidemiológicos mostram que as populações que nos seus primórdios dependiam da pecuária muito mais que da agricultura, e eram grandes consumidores de leite e laticínios em geral, apresentam menor prevalência de intolerância à lactose em relação àquelas que dependeram mais da agricultura para sobreviver, conforme está apresentado na Tabela 1 .

No geral, a prevalência da hipolactasia primária do adulto varia no mundo, sendo em torno de 5\% no nordeste da Europa próximo ao Mar do Norte, com a menor de todas encontrada na Dinamarca (4\%), na Grã-Bretanha (5\%), e na Suécia ( $1 \%$ a 7\%). A prevalência

Tabela 1- Prevalência de hipolactasia primária do adulto em diferentes populações

\begin{tabular}{|c|c|c|}
\hline Local & Prevalência (\%) & Método \\
\hline Alemanha $^{35}$ & 14,8 & Hidrogênio Expirado \\
\hline Áustria ${ }^{36}$ & 20,1 & Hidrogênio Expirado \\
\hline Brasil (brancos) $)^{37}$ & 57 & genético \\
\hline Brasil (crianças índias Terenas) ${ }^{38}$ & 89,3 após 4 anos & Hidrogênio Expirado \\
\hline Brasil (japoneses) 37 & 100 & genético \\
\hline Brasil (mulatos) $)^{37}$ & 57 & genético \\
\hline Brasil (negros) ${ }^{37}$ & 80 & genético \\
\hline China (Norte) ${ }^{39}$ & 87,3 & genético \\
\hline Estônia (ligados aos finlandeses) ${ }^{40}$ & 24,8 & genético \\
\hline França ${ }^{41}$ & 23,4 & Hidrogênio Expirado \\
\hline Hungria 26 & 37 & genético \\
\hline Índia (Norte) ${ }^{42}$ & 67,5 & genético \\
\hline Índia (sul) ${ }^{42}$ & 86,8 & genético \\
\hline Itália ${ }^{43}$ & 51(Norte) 71(Sicilia) & Hidrogênio Expirado \\
\hline Japão (adultos) $)^{44}$ & 89 & Hidrogênio Expirado \\
\hline Jordânia (beduínos) ${ }^{45}$ & 24 & Hidrogênio Expirado \\
\hline Jordânia (oeste) e Palestina ${ }^{45}$ & 75 & Hidrogênio Expirado \\
\hline Russia (Nordeste) ${ }^{46}$ & 35,6 & genético \\
\hline Sibéria (oeste, nativos Khants) ${ }^{47}$ & 94 & Tolerância/ glicemia \\
\hline Somalis ${ }^{48}$ & 76 & Hidrogênio Expirado \\
\hline Sudão (tribo Béja, pecuaristas) ${ }^{49}$ & 16,8 & Hidrogênio Expirado \\
\hline Sudão (tribo Nilotes, agricultores) ${ }^{49}$ & 74,5 & Hidrogênio Expirado \\
\hline Suécia (crianças caucasianas) ${ }^{50}$ & 10 & genético \\
\hline $\begin{array}{l}\text { Suécia (crianças } \\
\text { não-caucasianas) }\end{array}$ & 66 & genético \\
\hline Suécia (idoso caucasiano) ${ }^{50}$ & 5 & genético \\
\hline Tuaregues $^{51}$ & 12,7 & Hidrogênio Expirado \\
\hline Turquia $^{52}$ & 71,3 & Hidrogênio Expirado \\
\hline
\end{tabular}


da hipolactasia vai aumentando na direção do centro-sul da Europa para chegar próximo aos $100 \%$ na Ásia e Oriente Médio. ${ }^{53}$

$\mathrm{Na}$ África, com diferentes variantes alélicas, o genótipo de lactasia persistente foi mais frequente nos povos com tradição de pecuária em relação aos agricultores ${ }^{28}$. Embora no Brasil haja miscigenação racial com africanos, as evidências apontadas por DNA mitocondrial ${ }^{54}$ são de que os africanos trazidos para o Brasil vieram de regiões na África, onde o alelo LCT-13910T é prevalente. No nosso estudo ${ }^{37}$, o alelo LCT-13910T foi encontrado em $20 \%$ dos negros, sugerindo que tenha sido adquirido por miscigenação com Europeus ao longo dos séculos ao invés de ter sido trazido pelos fulanis africanos durante a escravidão.

\section{Fisiopatologia}

A lactose é encontrada apenas no leite materno, apresentando diferentes concentrações nos mamíferos. Em $100 \mathrm{~g}$ de leite de vaca desnatado existe $4,9 \mathrm{~g}$ de lactose $^{55}$ e em $100 \mathrm{ml}$ de leite humano, $7 \mathrm{~g}$ de lactose $^{56}$. O leite é o primeiro e único alimento do recém-nascido. Nos ratos e coelhos, a lactase não é detectada até alguns dias antes do nascimento, aumentando na fase tardia da gestação com pico logo após o nascimento. Já no intestino humano, os níveis de lactase são baixos até a $27^{a}-32^{a}$ semana de gestação, quando se elevam, rapidamente ${ }^{57}$, começando a cair por volta dos cinco anos de idade ${ }^{58}$. Desta forma, os bebês prematuros nascidos com 28 a 32 semanas de gestação têm atividade reduzida de lactase ${ }^{59}$, porém se forem de outra maneira saudáveis, o cólon pode recuperar os carboidratos não absorvidos, prevenindo a desnutrição e diarreia.

A enzima lactase hidrolisa a lactose em glicose e galactose que são absorvidas pela mucosa intestinal. A glicose entra para o pool de glicose do intestino, e a galactose é metabolizada no fígado para ser convertida em glicose, e entrar nesse pool. Caso a galactose não seja metabolizada no fígado, o é pelos eritrócitos, ou é eliminada na urina ${ }^{56}$. A concentração de enzima lactase na mucosa intestinal varia ${ }^{60}$, com atividade no duodeno $40 \%$ menor do que no jejuno ${ }^{61}$. A lactose, não sendo hidrolisada, não é absorvida no intestino delgado e passa rapidamente para o cólon. No cólon, a lactose é convertida em ácidos graxos de cadeia curta, gás carbônico e gás hidrogênio pelas bactérias da flora, produzindo acetato, butirato e propionato. Os ácidos graxos são absorvidos pela mucosa colônica, desta forma recuperando a lactose mal absorvida para utilização energética. Os gases, após absorção intestinal, são expirados pelo pulmão, servindo como ferramenta diagnóstica ${ }^{62}$. Esta fermentação da lactose pela flora bacteriana leva ao aumento do trânsito intestinal e da pressão intracolônica, podendo ocasionar dor abdominal e sensação de inchaço no abdome ${ }^{63}$. A acidificação do conteúdo colônico e o aumento da carga osmótica no íleo e cólon resultante da lactose não absorvida leva à grande secreção de eletrólitos e fluidos, além do aumento do trânsito intestinal, resultando em fezes amolecidas e diarreia60, 64. Apesar da falta de evidências, alguns autores acreditam que a absorção da lactose nos pacientes com hipolactasia poderia ser favorecida pela sua metabolização pela flora intestinal ${ }^{56}$.

\section{Intolerância à lactose congênita}

É herdada e autossômica recessiva, sendo uma condição extremamente grave. Caso não seja diagnosticada precocemente pode levar ao óbito. 0 recém- nascido apresenta diarreia líquida ao ser amamentado ou receber fórmulas, contendo lactose. 0 estudo genético realizado com 24 famílias finlandesas mostrou que se deve a mutações no gene $L C T$ que codifica a enzima lactase. A grande maioria dos pacientes ( $84 \%$ ) apresentou a mutação homozigota nonsense c.4170T $\rightarrow$ A (Y1390X), designada "Fin major", resultando em códon de parada de síntese proteica no éxon 9, e proteína truncada com 537 aminoácidos ${ }^{65}$. Na Finlândia, a incidência é de 1:60.000, sendo diagnosticada em 42 pacientes de 35 famílias de 1966 até 2007. Com dieta restritiva de lactose os sintomas desaparecem e esses recém-nascidos têm crescimento normal ${ }^{66}$. A diferença entre a hipolactasia primária do adulto e a intolerância à lactose congênita é molecular: na primeira, a enzima lactase é normal, mas diminui a expressão ao longo da vida; na segunda a enzima lactase está ausente, ou é truncada66.

\section{Métodos diagnósticos}

Os testes de tolerância à lactose são realizados com desafio, o paciente ingere de $25 \mathrm{~g}$ a $50 \mathrm{~g}$ de lactose e se avalia os sintomas por duas a três horas. A técnica mais difundida nos laboratórios de análises clínicas é a por curva glicêmica. Nesta técnica, é coletada a glicemia em jejum e depois é feita uma curva. Se o paciente absorver a lactose, a glicemia deve se elevar de 1,4 mmol/l ou mais ${ }^{22}$.

0 teste respiratório do hidrogênio expirado (realizado no Hospital das Clínicas da FMUSP) é considerado padrão-ouro para o diagnóstico de intolerância à lactose ${ }^{62,67}$, seguindo o primeiro Consenso de Roma para metodologia e indicações de teste respiratório do hidrogênio expirado para doenças gastrintestinais ${ }^{68}$. 0 paciente tem que fazer o preparo na véspera, só podendo ingerir dieta não fermentativa com restrição total de lactose, sem fumar (o cigarro aumenta o hidrogênio expirado). Deve também evitar antibióticos por um mês antes do exame (a presença da flora bacteriana é essencial para a produção do hidrogênio), não pode fazer exercícios físicos (aumentam o hidrogênio expirado) e tem que se apresentar para o exame com jejum de 10 a 12 horas, podendo ingerir água. A sensibilidade do exame é de $80 \%$ a $92,3 \%$ e a especificidade $100 \%$ com $25 \mathrm{~g}$ de lactose, desde que o preparo esteja correto ${ }^{18}{ }^{67}$. A flora bacteriana colônica fica com atividade diminuída em $\mathrm{pH}$ ácido (é esse o $\mathrm{pH}$ das fezes quando se dá a fermentação da lactose), podendo neste $\mathrm{pH}$ o exame ser falso-negativo ${ }^{62}$. 0 exame se baseia na produção de hidrogênio pela fermentação da lactose não absorvida: o hidrogênio entra na corrente sanguínea e é expirado pelo pulmão. 0 paciente sopra o basal, ingere a lactose, e depois sopra novamente após 60, 90, 120, 150 e 180 minutos, sendo considerado o exame positivo quando ocorre aumento de hidrogênio expirado em 20 ppm (partes por milhão) em relação ao valor basal. O paciente intolerante relata sintomas durante o exame, geralmente coincidindo com aumento do hidrogênio expirado. A leitura é feita em cromatógrafo gasoso específico para o hidrogênio ${ }^{18,67}$. Em aproximadamente $15 \%$ dos pacientes predomina flora produtora de metano: Methanobrevibacter smithii, o hidrogênio não se eleva ${ }^{62}$. Os pacientes com flora produtora de metano têm sintomas mais leves ${ }^{69}$.

Com a validação do polimorfismo $L C T-13910 \mathrm{C}>\mathrm{T}$, o exame genético passou a incorporar a rotina de exames de laboratório para diagnosticar hipolactasia. Na Europa o exame é feito com kit MutaReal Lactase Test que utiliza o PCR em tempo real70, e com kit GenoType LCT que se baseia na hibridização dos produtos de PCR de PCR multiplex com sondas selvagens e 
mutantes aderidas às tiras de nitrocelulose ${ }^{71}$. 0 kit que emprega hibridização reversa não sofre interferência dos polimorfismos africanos observados nos pacientes da raça negra imigrantes da África que vivem na Europa, e que provocam um desvio da curva de melting por PCR em tempo real, sendo considerado melhor ${ }^{72}$.

No Brasil foi implantada na rotina laboratorial do Hospital das Clínicas da FMUSP técnica que emprega PCR convencional e tratamento do produto de PCR com enzima de restrição (RFLPPCR). A concordância pelo Índice Kappa com teste respiratório do hidrogênio foi alta, apresentando 100\% de sensibilidade e $96 \%$ de especificidade ${ }^{18}$. A técnica de PCR-RFLP também é utilizada por outros autores na Europa 22,27,73.

O diagnóstico que se baseia em medir a atividade da enzima lactase em biópsia intestinal com kit Quick Lactase Test, embora também tenha apresentado sensibilidade e especificidade de $100 \%$ com teste genético de hipolactasia, é um exame invasivo ${ }^{74}$, dependendo de endoscopia para a coleta da biópsia da segunda porção do duodeno, e a atividade da enzima lactase no duodeno é menor que no jejuno ${ }^{61}$.

\section{Sintomas}

Os sintomas típicos incluem dor abdominal, sensação de inchaço no abdome, flatulência, diarreia, borborigmos e, particularmente nos jovens, vômitos. A dor abdominal pode ser em cólica e frequentemente é localizada na região periumbilical ou quadrante inferior. $\mathrm{O}$ borborigmo pode ser audível no exame físico e para o paciente. As fezes usualmente são volumosas, espumosas e aquosas. Uma característica importante é que estes indivíduos, mesmo com quadro de diarreia crônica, geralmente não perdem peso $^{75}$. Em alguns casos a motilidade gastrintestinal está diminuída e os indivíduos podem se apresentar com constipação, possivelmente como consequência da produção de metano ${ }^{64}$.

Alguns autores também acreditam que a intolerância à lactose seja responsável por diversos sintomas sistêmicos, como dores de cabeça e vertigens, perda de concentração, dificuldade de memória de curto prazo, dores musculares e articulares, cansaço intenso, alergias diversas, arritmia cardíaca, úlceras orais, dor de garganta e aumento da frequência de micção ${ }^{76-79}$. Na presença de sintomas sistêmicos, é preciso avaliar se de fato decorrem da intolerância à lactose, se são sintomas coincidentes ou se decorrem de alergia à proteína do leite de vaca (que afeta até $20 \%$ dos pacientes com sintomas sugestivos de intolerância à lactose) $)^{64,80}$.

Existe uma grande variabilidade de sintomas entre os pacientes com intolerância à lactose. Os fatores responsáveis por esta variabilidade incluem a osmolalidade e conteúdo de gordura do alimento no qual o açúcar é ingerido, o esvaziamento gástrico, a sensibilidade à distensão abdominal produzida pela carga osmótica da lactose não hidrolizada no intestino delgado superior, o trânsito intestinal e a resposta do cólon à carga de carboidrato. De uma maneira geral, os alimentos com alta osmolalidade e conteúdo de gordura diminuem o esvaziamento gástrico e reduzem a gravidade dos sintomas induzidos pela lactose. Para alguns autores, a flora fecal poderia se adaptar ao aumento crônico da lactose na dieta, reduzindo a incidência e intensidade dos sintomas após carga do carboidrato ${ }^{80}$, porém a melhora dos sintomas pode ocorrer independente da adaptação bacteriana $^{81}$, dificultando muitas vezes a suspeita diagnóstica de intolerância à lactose.

\section{Tratamento}

Nos pacientes com lactase não persistente, o tratamento é considerado exclusivamente naqueles com sintomas de intolerância à lactose. Inicialmente se recomenda evitar temporariamente leite e produtos lácteos da dieta para se obter remissão dos sintomas. Tal tarefa pode ser dificultada pela presença de alimentos com lactose não identificada na sua composição $0^{79}$. As maiores concentrações de lactose se encontram no leite e sorvete, enquanto que os queijos geralmente contêm quantidades menores (Tabela 2 ) $^{82}$.

Tabela 2 - Conteúdo de lactose no leite, produtos lácteos e alguns produtos manufaturados

\begin{tabular}{|c|c|c|}
\hline Alimento & Tipo & $\begin{array}{l}\text { Porcentagem } \\
\text { por peso }\end{array}$ \\
\hline \multirow{9}{*}{ Leite } & Desnatado * & 4,8 \\
\hline & Semi- desnatado* & 4,7 \\
\hline & Integral* & 4,6 \\
\hline & Condensado, integral, adoçado * & 12,3 \\
\hline & Pó desnatado * & 52,9 \\
\hline & Evaporado, integral* & 8,5 \\
\hline & Cabra & 4,4 \\
\hline & Humano & 7,2 \\
\hline & Ovelha & 5,1 \\
\hline \multirow{5}{*}{ Creme } & Único & \\
\hline & Duplo & 2,2 \\
\hline & Amargo & 1,7 \\
\hline & Creme fraiche & 2,7 \\
\hline & Imitação de creme, por exemplo, Elmlea, Tip Top, & $\begin{array}{c}2,1 \\
2,3-6,8\end{array}$ \\
\hline \multirow{15}{*}{ Queijo } & Brie / camembert & Traços \\
\hline & Cheddar & 0,1 \\
\hline & Requeijão & 4,4 \\
\hline & Requeijão, reduzido de gordura & 7,3 \\
\hline & Queijo Cottage & 3,1 \\
\hline & Queijo Cottage, reduzido de gordura & 3,3 \\
\hline & Queijo cremoso & Traços \\
\hline & Dinamarquês azul & Traços \\
\hline & Stilton & 0,1 \\
\hline & Edam / gouda & Traços \\
\hline & Feta & 1,4 \\
\hline & Queijo de cabra & 0,9 \\
\hline & Mussarela & Traços \\
\hline & Parmesão & 0,9 \\
\hline & Fatias de queijo processado & 5,0 \\
\hline \multirow{6}{*}{ logurte } & Natural & 4,7 \\
\hline & Fruta & 4,0 \\
\hline & logurte de beber & 4,0 \\
\hline & Fromage frais natural & 4,0 \\
\hline & Fromage frais de fruta & 3,0 \\
\hline & Tzatziki (com pepinos) & 0,3 \\
\hline \multirow{7}{*}{ Pudins } & Milkshake comum & 4,5 \\
\hline & Sorvete de baunilha não lácteo & 4,8 \\
\hline & Sorvete de baunilha lácteo & 5,2 \\
\hline & Sorvete de chocolate & 4,7 \\
\hline & Arroz doce & 3,9 \\
\hline & Creme feito com leite integral & 5,2 \\
\hline & Mousse de chocolate & 3,8 \\
\hline
\end{tabular}


A exclusão total e definitiva da lactose da dieta deve ser evitada, pois pode acarretar prejuízo nutricional de cálcio, fósforo e vitaminas, podendo estar associada com diminuição da densidade mineral óssea e fraturas ${ }^{83}$. Além disto, a maioria das pessoas intolerantes à lactose pode ingerir $12 \mathrm{~g} /$ dia de lactose (equivalente a um copo de leite) sem apresentar sintomas $\operatorname{adversos}^{84}$. A presença de lactose na preparação de comprimidos também já foi avaliada como potencial desencadeante de sintomas em pacientes com intolerância a lactose, porém sem diferenças nos níveis de hidrogênio expirado ou nos sintomas em comparação com placebo ${ }^{85}$.

Para evitar os prejuízos nutricionais decorrentes da exclusão total e definitiva da lactose da dieta, após exclusão inicial de lactose, geralmente é recomendada a sua reintrodução gradual de acordo com o limiar sintomático de cada indivíduo ${ }^{86}$. Nesta fase, algumas medidas não farmacológicas podem auxiliar na elevação deste limiar e contribuir para adaptação à lactose, como por exemplo, a sua ingestão junto com outros alimentos, o seu fracionamento ao longo do dia e o consumo de produtos lácteos fermentados e maturados ${ }^{86}$.

Caso estas medidas não funcionem para reduzir os sintomas de intolerância à lactose, medidas farmacológicas podem ser adotadas. A terapia de reposição enzimática com lactase exógena (+ $\beta$-galactosidase), obtida de leveduras ou fungos, constitui uma possível estratégia para a deficiência primária de lactose. Estes preparados comerciais de "lactase", quando adicionados a alimentos que contenham lactose ou ingeridos com refeições com lactose, são capazes de reduzir os sintomas e os valores de hidrogênio expirado em muitos indivíduos intolerantes à lactose. Entretanto, estes produtos não são capazes de hidrolisar completamente toda a lactose da dieta com resultados variáveis em cada paciente. As "lactases" exógenas estão disponíveis comercialmente na forma líquida e em cápsulas e tabletes, e possivelmente as diferentes preparações não são equivalentes. A enzima solúvel pode ser adicionada ao leite que é então refrigerado de um dia para o outro antes do uso (porém pouco prático para uso frequente). Existem também leites comerciais com baixo teor de lactose (pré-incubado com lactose já hidrolisada), porém não estão amplamente disponíveis em muitos restaurantes e lanchonetes. Apesar dos trabalhos enfatizarem a eficácia das formulações líquidas de "lactase" na melhora dos sintomas e na redução do hidrogênio expirado, a taxa real de eficácia apresenta resultados discrepantes, que decorrem do tipo de microorganismo utilizado, da contribuição da atividade residual da lactase da mucosa intestinal, e da dose de reposição utilizada $^{87-89}$. As preparações em cápsulas e tabletes utilizadas na hora da refeição são mais caras que o leite pré-hidrolisado, porém são eficazes, palatáveis, de fácil uso e praticamente sem efeitos colaterais, sendo uma boa alternativa para reposição enzimática nos pacientes intolerantes à lactose ${ }^{86,90,91}$. Os sintomas da síndrome do intestino irritável e da intolerância à lactose são muito parecidos e de fato, as duas condições podem coexistir ${ }^{92}$. Nos pacientes com intolerância à lactose que não apresentam melhora com dieta restrita de lactose, a presença de síndrome do intestino irritável subjacente deve ser aventada.

A ingestão de pró-bióticos contendo lactase teria o potencial de auxiliar na digestão da lactose nos indivíduos intolerantes, no entanto os estudos que avaliaram o benefício de sua utilização apresentaram resultados discordantes. Desta forma, no momento, os pró-bióticos têm papel incerto no manejo da intolerância à lactose ${ }^{86}$.

Como mencionado previamente, nos pacientes com intolerância à lactose e redução do consumo de leite e derivados, é importante avaliar se a ingestão diária de cálcio está adequada. Nos adolescentes e adultos jovens geralmente se recomenda cálcio na dieta de 1200 a 1500mg por dia. Já nos adultos a quantia diária varia de acordo com o sexo e presença de menopausa. O cálcio deve ser suplementado caso esteja insuficiente na dieta, com monitorização e suplementação se necessário também de vitamina $D$. logurtes com culturas vivas que contém beta-galactosidase endógena são uma fonte alternativa de calorias e cálcio e podem ser bem tolerado por muitos pacientes intolerantes. Porém os iogurtes que contém leite ou seus derivados que foram adicionados de volta após a fermentação podem produzir sintomas ${ }^{86}$.

\section{Conflito de interesse: Não há}

\section{SUMmarY}

\section{LACTOSE INTOLERANCE: CHANGING PARADIGMS DUE TO MOLECULAR BIOLOGY}

In most mammals, lactase activity declines on the intestinal wall after weaning, characterizing primary hypolactasia that provokes symptoms of lactose intolerance. The intensity of symptoms of distention, flatulence, abdominal pain and diarrhea varies, according to the amount of ingested lactose, and increases with age. Hypolactasia is genetically determined; nonetheless, a mutation occurred that had made a part of mankind tolerate milk in adulthood. Diagnosis is made by a tolerance test, using the lactose challenge. With the discovery made by the Finns of polymorphism associated with lactase persistence, mainly, in Northern Europe, the genetic test was incorporated as a more comfortable diagnostic tool for the intolerant. In Brazil, 43\% of Caucasian and Mulatto groups have lactase persistence allele, with hipolactasia more frequently found among Blacks and Japanese. However, in clinical practice people with hypolactasia may be advised to consume certain dairy products and food containing lactose without developing intolerance symptoms, whereas others will need a lactose restriction diet. [Rev Assoc Med Bras 2010; 56(2): 230-6]

KEY WORDS: Lactose intolerance. Lactose factors. Epidemiology. Molecular biology.

\section{REFERÊNCIAS}

1. Sahi T. Hypolactasia and lactase persistence. Historical review and the terminology. Scand J Gastroenterol. 1994;29 (Suppl) 202:1-6.

2. Enattah NS, Trudeau A, Pimenoff V, Maiuri L, Auricchio S, Greco L, et al. Evidence of still-ongoing convergence evolution of the lactase persistence T-13910 alleles in humans. Am J Hum Genet. 2007;81:615-25.

3. Wang Y, Harvey CB, Hollox EJ, Phillips AD, Poulter M, Clay P, et al. The genetically programmed down-regulation of lactase in children. Gastroenterology. 1998;114:1230-6.

4. Rana SV, Bhasin DK, Vinayak VK. Lactose hydrogen breath test in Giardia lamblia-positive patients. Dig Dis Sci. 2005;50:259-61.

5. Ojetti V, Nucera G, Migneco A, Gabrielli M, Lauritano C, Danese S, et al. High prevalence of Celiac disease in patients with lactose intolerance. Digestion. 2005; 71:106-10.

6. Tursi A, MD, Brandimarte G, Giorgetti GM, Elisei W. Transient Lactose malabsorption in patients affected by symptomatic uncomplicated diverticular disease of the colon. Dig Dis Sci. 2006;51:461-5. 
7. West A R, Oates PS. Decreased sucrase and lactase activity in iron deficiency is accompanied by reduced gene expression and upregulation of the transcriptional repressor PDX-1. Am J Physiol Gastrointest Liver Physiol. 2005;289:G1108-14.

8. Labayen I, Forga L, Gonzalez A, Lenoir-Wijnkoop I, Nutr R, Martinez JA. Relationship between lactose digestion, gastrointestinal transit time and symptoms in lactose malabsorbers after dairy consumption. Aliment Pharmacol Ther. 2001; 15:543-9

9. Mantei N, Villa M, Enzler T, Wacker H, Boll W, James P, et al. Complete primary structure of human and rabbit lactase-phlorizin hydrolase: implications for biosynthesis, membrane anchoring and evolution of the enzyme. EMBO J. 1988; 7:2705-13.

10. Naim HY, Sterchi EE, Lentze MJ. Biosynthesis and maturation of lactasephlorizin hydrolase in the human small intestinal epithelial cells. Biochem J. 1987;241:427-34.

11. Skovbjerg H, Sjôstrôm H, Nóren O. Purification and characterisation of amphiphilic lactase/phlorizin hydrolase from human small intestine. Eur J Biochem. $1981 ; 114: 653-66$

12. Kruse TA, Bolund L, Grzeschik KH, Ropers HH, Sjostrom H, Noren O, et al. The human lactase-phlorizin hydrolase gene is located on chromosome 2. FEBS Lett. 1988:240:123-6.

13. Boll W, Wagner P, Mantei N. Structure of the chromosomal gene and cDNAs coding for lactase-phlorizin hydrolase in humans with adult-type hypolactasia or persistence of lactase. Am J Hum Genet. 1991;48:889-90.

14. Enattah NS, Sahi T, Savilahti E, Terwilliger JD, Peltonen L, Jãverlã I. Identification of a variant associated with adult-type hypolactasia. Nat Genet. 2002;30:233-7.

15. Sahi T, Isokoski M, Jussila J, Launiala K, Pyörälä K. Recessive inheritance of adult-type lactose malabsorption. Lancet. 1973;302:823-6.

16. Bernardes-Silva CF, Pereira AC, da Mota GFA, Krieger JE, Laudanna AA. Lactase persistence/non-persistence variants, C/T_13910 and G/A_22018, as a diagnostic tool for lactose intolerance in IBS patients. Clin Chim Acta 2007;386:7-11.

17. Bulhões AC, Goldani HAS, Oliveira FS, Matte US, Mazzuca RB, Silveira TR. Correlation between lactose absorption and the C/T-13910 and G/A-22018 mutations of the lactase-phlorizin hydrolase (LCT) gene in adult-type hypolactasia. Braz J Med Biol Res. 2007;40:1441-6.

18. Mattar R, Monteiro MS, Villares CA, Santos AF, Carrilho FJ. Single nucleotide polymorphism $\mathrm{C} / \mathrm{T}_{-13910}$, located upstream of the lactase gene, associated with adult-type hypolactasia: validation for clinical practice. Clin Biochem. 2008; $41: 628-30$

19. Poulter M, Hollox E, Harvey CB, Mulcare C, Peuhkuri K, Kajander K, et al. The causal element for the lactase persistence/non-persistence polymorphism is located in a $1 \mathrm{Mb}$ region of linkage disequilibrium in Europeans. Ann Hum Genet. 2003;67:298-311.

20. Buning C, Genschel J, Jurga J, Fiedler T, Voderholzer W, Fiedler EM, et al. Introducing genetic testing for adult-type hypolactasia. Digestion. 2005; 71:245-50.

21. Hogenauer C, Hammer HF, Mellitzer K, Renner W, Krejs GJ, Toplak H. Evaluation of a new DNA test compared with the lactose hydrogen breath test for the diagnosis of lactase non-persistence. Eur J Gastroenterol Hepatol. 2005;17:371-6.

22. Ridefelt P, Hákansson LD. Lactose intolerance:lactose tolerance test versus genotyping. Scand J Gastroenterol. 2005;40:822-6.

23. Bodlaj G, Stöcher M, Hufnagl P, Hubmann R, Biesenbach G, Stekel H, et al. Genotyping of the Lactase-Phlorizin Hydrolase -13910 Polymorphism by LightCycler PCR and Implications for the Diagnosis of Lactose Intolerance. Clin Chem. 2006;52:148-51.

24. Szilagyi A, Malolepszy P, Hamard E, Xue X, Hilzenrat N, Ponniah M, et al. Comparison of a real-time polymerase chain reaction assay for lactase genetic polymorphism with standard indirect tests for lactose maldigestion. Clin Gastroenterol Hepatol. 2007;5:192-6.

25. Di Stefano M, Terulla V, Tana P, Mazzocchi S, Romero E, Corazza GR. Genetic test for lactase non-persistence and hydrogen breath test: is genotype better than phenotype to diagnose lactose malabsorption? Dig Liver Dis. 2009;41:474-9.

26. Nagy D, Bogácsi-Szabó E, Várkonyi Á, Csányi B, Czibula Á, Bede O, et al. Prevalence of adult-type hypolactasia as diagnosed with genetic and lactose hydrogen breath tests in Hungarians. Eur J Clin Nutr 2009; 63: 909-12.

27. Mulcare CA, Weale ME, Jones AL, Connell B, Zeitlyn D, Tarekegn A, et al. The allele of a single-nucleotide polymorphism $13.9 \mathrm{~kb}$ upstream of the lactase gene (LCT) $(\mathrm{C} 13.9 \mathrm{kbT})$ does not predict or cause the lactase-persistence phenotype in Africans. Am J Hum Genet. 2004;74:1102-10.

28. Tishkoff SA, Reed FA, Ranciaro A, Voight BF, Babbitt CC, Silverman JS, et al. Convergent adaptation of human lactase persistence in Africa and Europe. Nat Genet. 2007;39:1-10.

29. Olds LC, Sibley E. Lactase persistence DNA variant enhances lactase promoter activity in vitro: functional role as a cis regulatory element. Hum Mol Genet. 2003; $12: 2333-40$.
30. Kuokkanen M, Enattah NS, Oksanen A, Savilahti E, Orpana A, Järvelä I. Transcriptional regulation of the lactase-phlorizin hydrolase gene by polymorphisms associated with adult-type hipolactasia. Gut. 2003;52:647-52.

31. Burger J, Kirchner M, Bramanti B, Haak W, Thomas MG. Absence of the lactase-persistence-associated allele in early Neolithic Europeans. Proc Natl Acad Sci USA. 2007;104:3736-41.

32. CoelhoM, LuiselliD, BertorelleG, LopesAI, SeixasS, Destro-BisolG, et al. Microsatellite variation and evolution of human lactase persistence. Hum Genet. 2005; 117:329-39.

33. Wooding SP. Following the herd. Nat Genet. 2007;39:7-8

34. Beja-Pereira A, Luikart G, England PR, Bradley DG, Jann OC, Bertorelle G, et al. Gene-culture coevolution between cattle milk protein genes and human lactase genes. Nat Genet. 2003;35:311-3.

35. Flatz G, Howell JN, Doench J, Flatz SD. Distribution of physiological adult lactase phenotypes, lactose absorber and malabsorber, in Germany. Hum Genet. 1982;62:152-7.

36. Rosenkranz W, Hadorn B, Müller W, Heinz-Erian P, Hensen C, Flatz G. Distribution of human adult lactase phenotypes in the population of Austria. Hum Genet. 1982;62:158-61.

37. Mattar R, Monteiro MS, Villares CA, Santos AF, Silva JMK, Carrilho FJ. Frequency of LCT $-13910 \mathrm{C}>\mathrm{T}$ single nucleotide polymorphism associated with adult-type hypolactasia/lactase persistence among Brazilians of different ethnic groups. Nutr J. 2009;8:46.

38. Alves GMS, Moraes MB, Fagundes-Neto U. Nutritional status and breath hydrogen test with lactose and lactulose in Terena Indian children. J Pediatr. 2002;78:113-9.

39. Xu L, Sun H, Zhang X, Wang J, Sun D, Chen F, et al. The -22018 A allele matches the lactase persistence phenotype in northern Chinese populations. Scand J Gastroenterol. 2010;45:168-77.

40. Lember M, Torniainen S, Kull M, Kallikorm R, Saadla P, Rajasalu T, et al. Lactase non-persistence and milk consumption in Estonia. World J Gastroenterol. 2006;12:7329-31.

41. Cloarec D, Gouilloud S, Bornet F, Bruley des Varannes S, Bizais Y, Galmiche JP. Lactase deficiency and lactose intolerance-related symptoms in adult healthy subjects from western France. Gastroenterol Clin Biol. 1991;15:588-93.

42. Babu J, Kumar S, Babu P, Prasad JH, Ghoshal UC. Frequency of lactose malabsorption among healthy southern and northern Indian populations by genetic analysis and lactose hydrogen breath and tolerance tests. Am J Clin Nutr doi: 10.3945/ajen.2009.27946.

43. Burgio RG, Flatz G, Barbera C, Patané R, Bonner A, Cajozzo C, et al. Prevalence of primary adult lactose malabsorption and awareness of milk intolerance in Italy. Am J Clin Nutr 1984;39:100-4.

44. Nose O, lidaY, Kai H, Harada T, Ogawa M, Yabuuchi H. Breath hydrogen test for detecting lactose malabsorption in infants and children. Arch Dis Child 1979. 54:436-40.

45. Hijazi SS, Abulaban A, Ammarin Z, Flatz G. Distribution of adult lactase phenotypes in Bedouins and in urban and agricultural populations of Jordan. Trop Geogr Med 1983; 35:157-61.

46. Khabarova Y, Torniainen S, Nurmi H, Järvelä I, Isokoski M, Mattila K. Prevalence of lactase persistent/non-persistent genotypes and milk consumption in a young population in north-west Russia. World J Gastroenterol 2009; 15: 1849-53.

47. Lember M, Tamm A, Piirsoo A, Suurmaa K, Kermes K, Kermes R, et al. Lactose malabsorption in Khants in Western Siberia. Scand J Gastroenterol. 1995;30:225-7.

48. Ingram CJE, Raga TO, Tarekegn A, Browning SL, Elamin MF, Bekele E, et al. Multiple rare variants as a cause of a common phenotype: several different lactase persistence associated alleles in a single ethnic group. J Mol Evol. 2009;69:579-88.

49. Bayoumi RA, Flatz SD, Kühnau W, Flatz G. Beja and Nilotes: nomadic pastoralist groups in the Sudan with opposite distributions of the adult lactase phenotypes. Am J Phys Anthropol. 1982;58:173-8.

50. Almon R, Engfeldt P, Tysk C, Sjöström M, Nilsson TK. Prevalence and trends in adult-type hypolactasia in different age cohorts in Central Sweden diagnosed by genotyping for the adult-type hypolactasia-linked LCT-13910C > T mutation. Scand J Gastroenterol. 2007;42:165-70.

51. Flatz G, Schildge C,Sekou H. Distribution of Adult Lactase Phenotypes in the Tuareg of Niger. Am J Hum Genet .1986;38:515-20.

52. Flatz G, Henze HJ, Palabiyikoglu E, Dagalp K, Türkkan T. Distribution of the adult lactase phenotypes in Turkey. Trop Geogr Med. 1986;38:255-8.

53. Sahi T. Genetics and epidemiology of adult-type hypolactasia. Scand J Gastroenterol. 1994;29:7-20.

54. Gonçalves VF, Carvalho CMB, Bortolini MC, Bydlowski SP, Pena SDJ. The Phylogeography of African Brazilians. Hum Hered. 2008;65:23-32.

55. Lummela N, Kekkonen RA, Jauhiainen T, Pilvi TK, Tuure T, Järvenpää S, et al. Effects of a fibre-enriched milk drink on insulin and glucose levels in healthy subjects. Nutr J. 2009;8:45.

56. Arola H, Tamm A. Metabolism of lactose in the human body. Scand J Gastroenterol. 1994;29:21-5. 
57. Montgomery RK, Büller HA, Rings EHHM, Grand RJ. Lactose intolerance and the genetic regulation of intestinal lactase-phlorizin hydrolase. FASEB J. 1991:5:2824-32.

58. Lebenthal E, Antonowicz I, Shwachman H. Correlation of lactase activity, lactose tolerance and milk consumption in different age groups. Am J Clin Nutr. 1975;28:595-600.

59. Buller, HA, Grand, RJ. Lactose intolerance. In: Creger, WP, Coggins, CH, Hancock, EW. Annual Review of Medicine: selected topics in the clinical sciences. Palo Alto: Annual Reviews Inc; 1990. p.141.

60. Robayo-Torres CC, Quezada-Calvillo R, Nichols BL. Dissaccharide digestion: clinical and molecular aspects. Clin Gastroenterol Hepatol. 2006;4:276-87.

61. Langman JM, Rowland R. Activity of duodenal dissaccharidases in relation to normal and abnormal mucosal morphology. J Clin Pathol. 1990;43:537-40.

62. Romagnuolo J, Schiller D, Bailey RJ. Using breath tests wisely in a gastroenterology practice: an evidence-based review of indications and pitfalls in interpretation. Am J Gastroenterol. 2002;97:1113-26.

63. He T, Priebe MG, Harmsen HJ, Stellaard F, Sun X, Welling GW, et al. Colonic fermentation may play a role in lactose intolerance in humans. J Nutr. 2006; 136:58-63.

64. Lomer MCE, Parkes GC, Sanderson JD. Review article: lactose intolerance in clinical practice - myths and realities. Aliment Pharmacol Ther 2008;27:93-103.

65. Kuokkanen M, Kokkonen J, Enattah NS, Ylisaukko-oja T, Komu H, Varilo T, et al. Mutations in the translated region of the lactase gene $(L C T)$ underlie congenital lactase deficiency. Am J Hum Genet. 2006;78:339-44.

66. Robayo-Torres CC, Nichols BL. Molecular differentiation of congenital lactase deficiency from adult- type hypolactasia. Nutr Rev. 2007;65:95-8.

67. Mattar R. Investigação laboratorial em gastroenterologia. In: Martins MA, Carrilho FJ, Alves, VAF, Castilho EA, Cerri GG, Wen CL. Clínica médica. Barueri: Manole; 2009. Vol. 4, p.28-39: Doenças do aparelho digestivo e doenças nutricionais.

68. Gasbarrini A, Corazza GR, Gasbarrini G, Montalto M, Di Stefano M, Basilisco $\mathrm{G}$, et al. Methodology and indications of $\mathrm{H} 2$-breath testing in gastrointestinal diseases: the Rome Consensus Conference. Aliment Pharmacol Ther. 2009;29(Suppl.1):1-49.

69. Vernia P, Di Camilo M, Marinaro V, Caprilli R. Effect of predominant methanogenic flora on the outcome of lactose breath test in irritable bowel syndrome patients. Eur J Clin Nutr. 2003;57:1116-9.

70. Tag CG, Schifflers M-C, Mohnen M, Gressner AM, Weiskirchen R. A novel proximal $13914 \mathrm{G}>\mathrm{A}$ base replacement in the vicinity of the common$13910 \mathrm{~T} / \mathrm{C}$ lactase gene variation results in an atypical LightCycler melting curve in testing with the MutaREAL lactase test. Clin Chem. 2007;53:146-8.

71. Di Stefano M, Terulla V, Tana P, Mazzocchi S, Romero E, Corazza GR. Genetic test for lactase non-persistence and hydrogen breath test: is genotype better than phenotype to diagnose lactose malabsorption? Dig Liver Dis. 2009:41:474-9.

72. Tag CG, Oberkanins C, Kriegshäuser G, Ingram CJE, Swallow DM, Gressner $A M$, et al. Evaluation of a novel reverse-hybridization StripAssay for typing DNA variants useful in diagnosis of adult-type hipolactasia. Clin Chim Acta. 2008:392:58-62.

73. Büning $C$, Ockenga J, Krüger $S$, Jurga J, Baier $P$, Dignass $A$, et al. The C/C-13910 and G/G-22018 genotypes for adult-type hypolactasia are not associated with Inflammatory Bowel Disease. Scand J Gastroenterol. 2003;38:538-42

74. Kuokkanen M, Myllyniemi M, Vauhkonen M, Helske T, Kääriäinen I, Karesvuori $\mathrm{S}$, et al. A biopsy-based quick test in the diagnosis of duodenal hypolactasia in upper gastrointestinal endoscopy. Endoscopy. 2006;38:708-12.

75. Semrad CE, Powell DW. Approach to the patient with diarrhea and malabsorption. In: Goldman L, Ausiello D, editors. Cecil medicine. $23^{\text {rd }}$ ed. Philadelphia: Saunders; 2008. p.1019-42.
76. Treudler R, Tebbe B, Steinhoff M, Orfanos CE. Familial aquagenic urticaria associated with familial lactose intolerance. J Am Acad Dermatol. 2002;47:611-13.

77. Matthews SB, Campbell AK. When sugar is not so sweet. Lancet. 2000;355:1309.

78. Matthews SB, Campbell AK. Neuromuscular symptoms associated with lactose intolerance. Lancet. 2000;356:511.

79. Matthews SB, Waud JP, Roberts AG. Systemic lactose intolerance: a new perspective on an old problem. Postgrad Med J. 2005;81:167-73.

80. Hertzler, SR, Savaiano, DA. Colonic adaptation to daily lactose feeding in lactose maldigesters reduces lactose intolerance. Am J Clin Nutr. 1996;64:232.

81. Briet F, Pochart P, Marteau P, Flourie B, Arrigoni E, Rambaud JC. Improved clinical tolerance to chronic lactose ingestion in subjects with lactose intolerance: A placebo effect? Gut. 1997;41:632-5.

82. Holland B, Welch AA, Unwin ID, Buss DH, Paul AA. Southgate DAT. In: Holland B, Welch AA, Unwin ID, Buss DH, Paul AA, Southgate DAT. McCance and Widdowson's, the composition of foods. 5th ed. London: The Royal Society of Chemistry; 1991.

83. Di Stefano M, Veneto G, Malservisi S, Cecchetti L, Minguzzi L, Strocchi A, et al. Lactose malabsorption and intolerance and peak bone mass. Gastroenterology. 2002;122:1793-9.

84. Vonk RJ, Priebe MG, Koetse HA, Stellaard F, Lenoir-Wijnkoop I, Antoine JM, et al. Lactose intolerance: analysis of underlying factors. Eur J Clin Invest. 2003;33:70-5

85. Montalto M, Gallo A, Santoro L, DOnofrio F, Curigliano V, Covino M, et al. Lowdose lactose in drugs neither increases breath hydrogen excretion nor causes gastrointestinal symptoms. Aliment Pharmacol Ther. 2008;28:1003-12.

86. Montalto M, Curigliano V, Santoro L, Vastola M, Cammarota G, Manna R, et al. Management and treatment of lactose malabsorption. World J Gastroenterol. 2006;12:187-91.

87. Rosado JL, Solomons NW, Lisker R, Bourges H. Enzyme replacement therapy for primary adult lactase deficiency. Effective reduction of lactose malabsorption and milk intolerance by direct addition of beta-galactosidase to milk at mealtime. Gastroenterology. 1984;87:1072-82.

88. Solomons NW, Guerrero AM, Torun B. Dietary manipulation of postprandial colonic lactose fermentation: II. Addition of exogenous, microbial betagalactosidases at meal time. Am J Clin Nutr. 1985;41:209-21.

89. Lin MY, Dipalma JA, Martini MC, Gross CJ, Harlander SK, Savaiano DA. Comparative effects of exogenous lactase (beta-galactosidase) preparations on in vivo lactose digestion. Dig Dis Sci. 1993;38:2022-7.

90. Suarez FL, Savaiano DA, Levitt MD. Review article: the treatment of lactose intolerance. Aliment Pharmacol Ther. 1995;9:589-97.

91. Flood MT, Kondo M. Toxicity evaluation of a beta-galactosidase preparation produced by Penicillium multicolor. Regul Toxicol Pharmacol. 2004:40:281-92.

92. Heizer WD, Southern S, Mcgovern S. The role of diet in symptoms of irritable bowel syndrome in adults: a narrative review. J Am Diet Assoc. 2009;109:1204-14.

Artigo recebido: 16/12/09 Aceito para publicação: 23/01/10 